\title{
Temperature-sensitive PSII: a novel approach for sustained photosynthetic hydrogen production
}

\author{
Vinzenz Bayro-Kaiser ${ }^{1} \cdot$ Nathan Nelson $^{1}$
}

Received: 8 December 2015/Accepted: 11 February 2016/Published online: 7 March 2016

(C) The Author(s) 2016. This article is published with open access at Springerlink.com

\begin{abstract}
The need for energy and the associated burden are ever growing. It is crucial to develop new technologies for generating clean and efficient energy for society to avoid upcoming energetic and environmental crises. Sunlight is the most abundant source of energy on the planet. Consequently, it has captured our interest. Certain microalgae possess the ability to capture solar energy and transfer it to the energy carrier, $\mathrm{H}_{2} . \mathrm{H}_{2}$ is a valuable fuel, because its combustion produces only one by-product: water. However, the establishment of an efficient biophotolytic $\mathrm{H}_{2}$ production system is hindered by three main obstacles: (1) the hydrogen-evolving enzyme, [FeFe]-hydrogenase, is highly sensitive to oxygen; (2) energy conversion efficiencies are not economically viable; and (3) hydrogen-producing organisms are sensitive to stressful conditions in large-scale production systems. This study aimed to circumvent the oxygen sensitivity of this process with a cyclic hydrogen production system. This approach required a mutant that responded to high temperatures by reducing oxygen evolution. To that end, we randomly mutagenized the green microalgae, Chlamydomonas reinhardtii, to generate mutants that exhibited temperaturesensitive photoautotrophic growth. The selected mutants were further characterized by their ability to evolve oxygen and hydrogen at 25 and $37{ }^{\circ} \mathrm{C}$. We identified four candidate mutants for this project. We characterized these mutants with PSII fluorescence, P700 absorbance, and immunoblotting analyses. Finally, we demonstrated that these mutants could function in a prototype hydrogen-
\end{abstract}

Nathan Nelson

nelson@post.tau.ac.il

1 Department of Biochemistry, The George S. Wise Faculty of Life Sciences, Tel Aviv University, 69978 Tel Aviv, Israel producing bioreactor. These mutant microalgae represent a novel approach for sustained hydrogen production.

Keywords Photosynthesis - Hydrogen production · Sustainability - Temperature-sensitive mutants . Photosystem II · Chlamydomonas reinhardtii

\section{Introduction}

Photosynthesis is one of the most important life-sustaining reactions on our planet. It provides the energy required for the survival of all life forms, and it underlies the accumulation of fossil fuels, the main source of energy for sustaining modern human lifestyles (Barber 2004; Nelson and Yocum 2006). The evolution of photosynthesis is a prime example of a highly complex process that enabled the development of current life forms on earth. Photosynthesis was established on earth more than 3.5 billion years ago under anaerobic conditions (Falkowski and Isozaki 2008; Blankenship and Hartman 1998; Bendall et al. 2008). Oxygenic photosynthesis, which evolved shortly thereafter, used light energy and water to produce reducing equivalents. These equivalents could be used as chemical energy, and oxygen evolved as a by-product. About 1.5 billion years passed before metal compounds in the oceans and on the earth's surface were oxidized, which allowed oxygen to accumulate in the atmosphere (Nelson 2011). During that period, oxygenic photosynthesis was limited by a shortage of oxidized electron acceptors, which are plentiful on the current earth surface. Arguably, protons served as a sink for the excess electrons, resulting in hydrogen molecules, most of which left earth, due to their light atomic weight, which allowed it to achieve escape velocity $(11.2 \mathrm{~km} / \mathrm{s})$ at normal temperatures (Nelson 2011). Although this 
mechanism may not seem necessary in the present oxygenrich atmosphere, it was discovered that some bacteria, microalgae, and cyanobacteria exhibited light-dependent hydrogen evolution (Gest and Kamen 1949; Lambert and Smith 1977; Amon et al. 1961). The enzyme responsible for this reaction uses electrons delivered by the photosynthetic electron transport chain (ETC), and this enzyme is highly sensitive to oxygen. Therefore, the reaction occurs strictly under anoxic conditions in the light. Within this framework, the notion of exploiting the photosynthetic apparatus to transform the most abundant energy source on our planet into the cleanest fuel is quite tempting (Kruse and Hankamer 2010).

Under strict anaerobic conditions, certain microalgae express the HYDA gene. This gene encodes a highly active, but oxygen-sensitive, [FeFe]-hydrogenase, which catalyzes the reversible reduction of protons to form $\mathrm{H}_{2}$ (Happe and Kaminski 2002). This hydrogenase is localized in the chloroplast stroma, coupled to the reducing site of the photosynthetic ETC (Winkler et al. 2009). The photosynthetic ETC is composed of several enzymes, most of which are embedded in the thylakoid membranes. The ETC is powered by light, which excites the reaction centers of Photosystem II (PSII) and Photosystem I (PSI). Light excitation of PSII results in the oxidation of water and the released electrons are transferred to PSI. When PSI is excited by light, these electrons are used to reduce ferredoxin (Nelson and Yocum 2006; Nelson and Ben-Shem 2004; Amunts and Nelson 2009; Nelson and Junge 2015). Ferredoxin may then supply reducing equivalents to $[\mathrm{FeFe}]$-hydrogenase, which reduces protons to form $\mathrm{H}_{2}$ (Florin et al. 2001). PSI is indispensable for ferredoxin-dependent hydrogen production. Hydrogen production mainly takes place in the light (Redding et al. 1999). However, in the light, the hydrogenase is rapidly and irreversibly inactivated by PSII-induced $\mathrm{O}_{2}$ production (Ghirardi et al. 1997; Yacoby et al. 2011). PSII is not directly essential for hydrogen production, because electrons from other sources can be fed into the ETC by transferring them to the PQ-pool (Stuart and Gaffron 1972; Melis et al. 2000). However, the main non-photosynthetic electron source for reducing the PQ-pool is starch, which is produced by PSII-dependent photosynthesis (Hemschemeier et al. 2008).

Before we can even consider the option of exploiting this mechanism for mass hydrogen production, we must overcome three major challenges. The first challenge is to spatially separate oxygen and hydrogen production to prevent combustion to water and to promote the required anoxic conditions. The second challenge is to convert light energy efficiently, to ensure that the process is economically viable. The third challenge is the inevitable sensitivity of the target organism to external agents, such as chemicals, viruses, and stress conditions.

In an attempt to circumvent the oxygen sensitivity of photosynthetic hydrogen production, a system has been proposed that requires an organism with a temperaturesensitive PSII (Mazor et al. 2012). At low temperatures, this organism would perform regular oxygenic photosynthesis and accumulate starch; at high temperatures, PSII would be inactive, and anaerobic conditions would be sustained in the light. Then, hydrogen production could take place, provided that the photosynthetic electron flow is maintained by pumping electrons from starch through the plastoquinone. This kind of organism would be cycled between two growth chambers, with different temperatures; hence, it would alternate between aerobic biomass production and anaerobic hydrogen production.

In this study, we used random mutagenesis on the model organism, $C$. reinhardtii, to generate an organism with a temperature-sensitive PSII. Mutated $C$. reinhardtii cells with the required phenotype were selected, identified, and characterized. These mutants were able to produce hydrogen, and we constructed a prototype photosynthetic hydrogen-producing bioreactor.

\section{Materials and methods}

\section{Random mutagenesis and screening}

C. reinhardtii wild-type, strain $1 \mathrm{~A}+$, was grown in liquid TAP medium (Harris 2008) to mid-log phase. Then, 200-500 cells were plated on TAP agar plates. The plates were exposed to UV radiation for a time period sufficient to kill 80-90\% of cells. After UV exposure, plates were maintained in the light at $25^{\circ} \mathrm{C}$, until the surviving cells grew to visible colonies. Single colonies were resuspended in water and re-plated on three TAP agar plates. Of these, two plates lacked acetate (photoautotrophic) and were maintained in light, one at $25^{\circ} \mathrm{C}$ and the other at $35^{\circ} \mathrm{C}$; the third plate was maintained in light at $35^{\circ} \mathrm{C}$. Strains that grew photoautotrophically at low temperature and only grew heterotrophically at high temperature were identified as temperature-sensitive photoautotrophic (TSP) mutants.

\section{Physiological measurements}

C. reinhardtii wild-type strain and TSP mutants were grown photoheterotrophically in liquid TAP medium, on a shaker, with constant illumination $\left(70 \mu \mathrm{mol} \mathrm{m} \mathrm{m}^{-2} \mathrm{~s}^{-1}\right)$, and at either 25 or $37^{\circ} \mathrm{C}$. Cell density was monitored by measuring absorbance at $730 \mathrm{~nm}$. The chlorophyll content was determined by acetone solubilization, as previously described (Arnon 1949). 
The net oxygen exchange rate (OER) and oxygen respiration rate were measured with a Clark-type oxygen electrode (Oxygraph plus, Hansatech). An aliquot of cell culture $\left(2 \mathrm{ml}\right.$ at a cell density of $\left.\mathrm{OD}_{730 \mathrm{~nm}}=0.5-0.7\right)$ was transferred to the measuring chamber, and changes in oxygen concentration were measured over time, both at a light intensity of $300 \mu \mathrm{mol} \mathrm{m} \mathrm{m}^{-2} \mathrm{~s}^{-1}$ and in the dark. At the same time, the chlorophyll concentration in the cell culture was determined. The oxygen concentration changes were normalized to the chlorophyll concentration.

The maximum quantum efficiency of PSII (Fv/Fm) was calculated from in vivo chlorophyll fluorescence measurements, as previously described (Baker 2008). A JTS-10 spectrophotometer was used. An aliquot of cell culture ( $2 \mathrm{ml}$ at a cell density of $\left.\mathrm{OD}_{730 \mathrm{~nm}}=0.5-0.7\right)$ was incubated in the dark for 5 min and transferred into a measuring cuvette. Chlorophyll was excited with a low-intensity light $\left(2 \mu \mathrm{mol} \mathrm{m} \mathrm{m}^{-2} \mathrm{~s}^{-1}\right)$ at $520 \mathrm{~nm}$, and fluorescence emission was measured at $\lambda>650 \mathrm{~nm}$. This was performed before and immediately after illuminating the sample with a highintensity light pulse of $2900 \mu \mathrm{mol} \mathrm{m}^{-2} \mathrm{~s}^{-1}$ at $520 \mathrm{~nm}$ for $250 \mathrm{~ms}$.

The amount of P700 and the oxidized P700 half-life were calculated from in vivo oxidized P700 absorbance measurements with a JTS-10 spectrophotometer. An aliquot of cell culture $(1 \mathrm{ml}$ at a cell density of $\mathrm{OD}_{730 \mathrm{~nm}}=0.5-0.7$ ) was incubated in the dark for $5 \mathrm{~min}$ and was transferred into a measuring cuvette. Absorbances of oxidized P700 were measured at $705 \mathrm{~nm}$, first with no actinic light, then after illuminating the sample with a highintensity light pulse of $3000 \mu \mathrm{mol} \mathrm{m}{ }^{-2} \mathrm{~s}^{-1}$. The amount of P700 was expressed as the difference in absorbance measured before and immediately after the saturation pulse, normalized by the chlorophyll content. The development of absorbance after the saturation pulse was used to calculate the half-life.

\section{Immunoblotting}

To prepare crude protein extracts, $1.5 \mathrm{ml}$ of cell culture was centrifuged ( $2 \mathrm{~min}, 14,000 \times \mathrm{g}$, room temperature) and the pellet was resuspended in Laemmli protein buffer (65.8 mM Tris-HCl, pH 6.8, $2.1 \%$ SDS, $26.3 \%$ (w/v) glycerol, $0.01 \%$ bromophenol blue). The final concentration corresponded to a chlorophyll content of $0.14 \mu \mathrm{g} \mu \mathrm{l}^{-1}$. Extracts were incubated at $65{ }^{\circ} \mathrm{C}$ for $30 \mathrm{~min}$, then centrifuged ( $2 \mathrm{~min}, 14,000 \times \mathrm{g}$, room temperature) to pellet the cell debris. Supernatants were loaded onto a sodium dodecyl sulfate polyacrylamide gel electrophoresis (SDSPAGE) to separate the proteins. The polyacrylamide gel comprised 5 and $16 \%$ acrylamide sections for protein collection and separation, respectively. The separated proteins were transferred to a nitrocellulose membrane with a semi-dry transfer cell (Trans-Blot SD from Bio$\mathrm{Rad})$, according to the manufacturer's instructions. The membrane was incubated for $1 \mathrm{~h}$ at room temperature in blocking buffer $(20 \mathrm{mM}$ Tris-HCl, $\mathrm{pH} 7.4,0.5 \mathrm{M} \mathrm{NaCl}$, $0.1 \%$ Tween-20, $5 \%(\mathrm{w} / \mathrm{v})$ skim milk powder), then overnight at $4{ }^{\circ} \mathrm{C}$ in blocking buffer with a primary antibody, diluted 1:4000 (Liveanu et al. 1986). Primary antibodies were rabbit anti-D1 (reaction center protein) and rabbit anti-CP43 (antenna chlorophyll-binding protein). Next, the membrane was washed 3 times for $15 \mathrm{~min}$ in blocking buffer, then incubated for $1 \mathrm{~h}$ at room temperature in blocking buffer with the secondary antibody (peroxidase-conjugated goat-anti-rabbit $\mathrm{IgG}$ from Jackson ImmunoResearch), diluted 1:5000. This was followed by a second wash repeated 3 times for 15 min with blocking buffer, then once for 15 min with blocking buffer that lacked powdered milk. The peroxidase substrate (Western lightning Plus-ECL from Perkin Elmer) was added to the secondary antibody, according to the manufacturer's instructions. The luminescent signal was detected on X-ray film. The primary antibodies were raised in rabbits injected with denatured D1 and CP43 proteins, which were electroeluted from PAGE gels (Liveanu et al. 1986).

\section{Hydrogen evolution assay}

An aliquot of cell culture $(15 \mathrm{ml}$ at a cell density of $\left.\mathrm{OD}_{730 \mathrm{~nm}}=0.5-0.7\right)$, with or without addition of a PSII inhibitor (10 mM DCMU), was transferred into a $25-\mathrm{ml}$ glass bottle and sealed with a rubber cap. The bottle was flushed with argon for $1 \mathrm{~min}$ to deplete it of air, and then it was placed on a shaker in the light or in the dark. At different time points, $500 \mu \mathrm{l}$ of the gas in the headspace was collected with a syringe and analyzed with a gas chromatograph (5890 Series II, Hewlett Packard). Simultaneously, $200 \mu \mathrm{l}$ of cell culture was also collected with a syringe to determine the chlorophyll content. Over the period of hydrogen production, the average hydrogen production rates normalized to chlorophyll were calculated.

\section{Results}

C. reinhardtii cells were exposed to UV irradiation to induce random mutagenesis. Colonies $(n=12,000)$ grown from single cells were screened for temperature-sensitive photoautotrophic (TSP) mutants. We identified 157 TSP mutants, which were further characterized with physiological and biomolecular assays. Of these, four were selected as the most suitable: TSP1, TSP2, TSP3, and TSP4. The phenotypes of these mutants resembled the wild-type microalgae at $25{ }^{\circ} \mathrm{C}$, but they differed from wild type at $37{ }^{\circ} \mathrm{C}$. The oxygen exchange rates (OERs) of wild- 
type and mutant cultures were measured in the dark $(0$ $\left.\mu \mathrm{E} \mathrm{m}^{-2} \mathrm{~s}^{-1}\right)$ and in the light $\left(300 \mu \mathrm{E} \mathrm{m}^{-} \mathrm{s}^{-1}\right)$ during $37{ }^{\circ} \mathrm{C}$ incubations (Fig. 1). Over the course of $36 \mathrm{~h}$, we measured oxygen respiration in the dark. In wild-type cultures, oxygen respiration decreased steadily from 99 to $55 \mathrm{nmol} \mu \mathrm{g}^{-1} \mathrm{~h}^{-1}$. All the mutant cultures showed similar declines in oxygen respiration in the dark. In the light, the net oxygen evolution in wild-type cultures also decreased over the course of $36 \mathrm{~h}$, from 120 to $49 \mathrm{nmol} \mathrm{gg}^{-1} \mathrm{~h}^{-1}$. However, in the mutant cultures, oxygen evolution also declined, but at a significantly faster rate compared to wildtype cultures. The values fell below $0 \mathrm{nmol} \mu \mathrm{g}^{-1} \mathrm{~h}^{-1}$ after $24 \mathrm{~h}$ for TSP1, TSP2, and TSP3, and between 6 and $12 \mathrm{~h}$ for TSP4. Furthermore, TSP4 OERs were the same in the dark and in the light after 12-h incubations.

To determine whether an absent PSII reaction center was responsible for the observed OER phenotype in mutants (Fig. 2a), we measured the maximum quantum efficiency of PSII with fluorescence spectroscopy over a 24-h period, during a $37{ }^{\circ} \mathrm{C}$ incubation. The PSII efficiency in TSP1 and TSP4 decreased by 50 and $100 \%$, respectively, after $12 \mathrm{~h}$, and it remained stable thereafter. The PSII efficiency in TSP3 remained unaltered during the first $18 \mathrm{~h}$ and decreased by $25 \%$ only after $24 \mathrm{~h}$. The PSII efficiency in TSP2 remained unaltered over the entire period of incubation. Next, we measured P700 oxidation in response to a high-intensity light pulse, and we measured
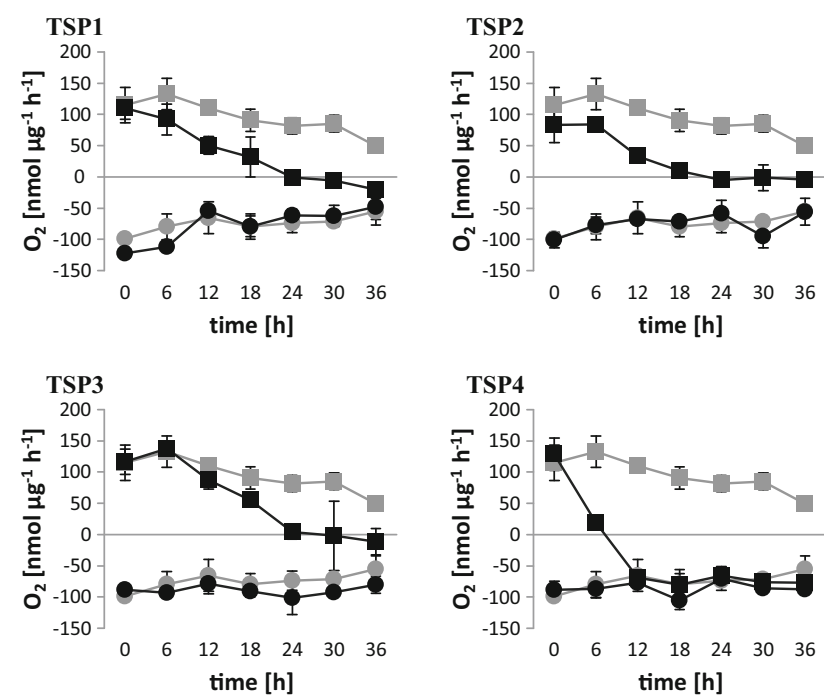

Fig. 1 Oxygen exchange rates in C. reinhardtii wild-type and TSP mutants at $37{ }^{\circ} \mathrm{C}$. All cultures were initially grown at $25^{\circ} \mathrm{C}$. In all charts, the gray curves correspond to the wild type; the black curves correspond to TSP1, TSP2, TSP3, and TSP4, as indicated. The light intensity was $60-70 \mu \mathrm{E} \mathrm{m}^{-2} \mathrm{~s}^{-1}$ during growth and during incubation at $37^{\circ} \mathrm{C}$. Measurements were performed in the dark (light intensity $=0 ;$ circles $)$ and at a light intensity of $300 \mu \mathrm{E} \mathrm{m}^{-2} \mathrm{~s}^{-1}$ (squares). Data represent the mean $\pm \mathrm{SD}$ of three independent experiments
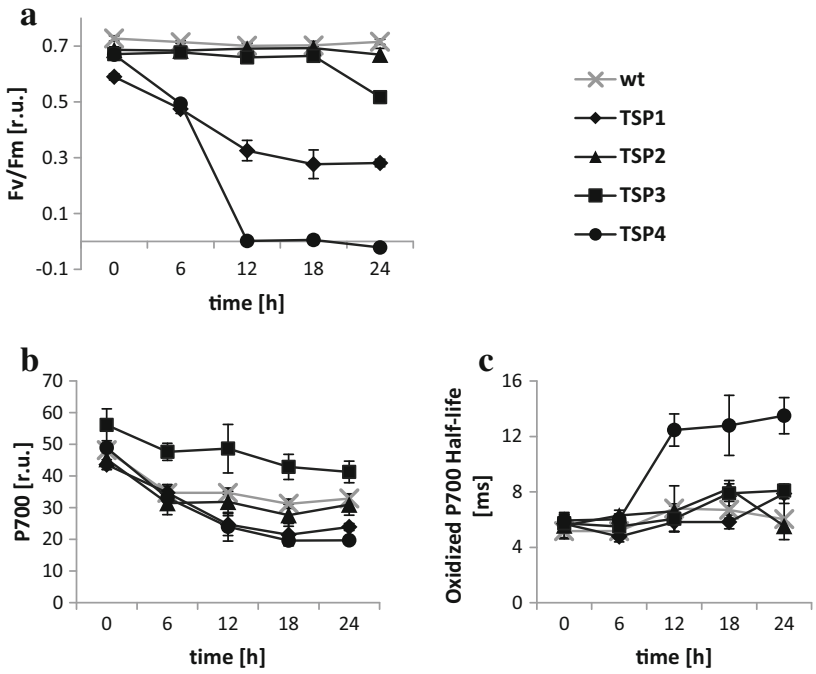

Fig. 2 PSII and PSI reactions in C. reinhardtii wild-type and TSP mutants at $37{ }^{\circ} \mathbf{C}$. a Maximum quantum efficiency of PSII, $\mathbf{b}$ amount of P700, and c oxidized P700 half-life; wild type is shown with gray crosses; mutant strains: TSP1 (diamonds), TSP2 (triangles), TSP3 (squares), and TSP4 (circles). All cells were incubated at $37^{\circ} \mathrm{C}$ after growth at $25{ }^{\circ} \mathrm{C}$. The light intensity was $60-70 \mu \mathrm{E} \mathrm{m}^{-2} \mathrm{~s}^{-1}$ during growth and during incubation at $37^{\circ} \mathrm{C}$. Data represent the mean $\pm \mathrm{SD}$ of three independent experiments

the subsequent reduction by determining light absorbance at $705 \mathrm{~nm}$ (Fig. 2b, c). At 12, 18, and $24 \mathrm{~h}$ of incubation at $37{ }^{\circ} \mathrm{C}$, TSP1 and TSP4 had $30 \%$ less P700 than wild-type algae. In contrast, TSP3 had 20 to $40 \%$ greater amounts of P700 than wild-type microalgae over the whole period of incubation. The amount of P700 in TSP2 was similar to that in wild-type microalgae. The half-life of the fully oxidized P700 in the wild type was 5-8 ms, measured over the entire study period at $37^{\circ} \mathrm{C}$. Furthermore, TSP1, TSP2, and TSP3 showed half-lives similar to that of wild-type algae. However, TSP4 had a longer oxidized P700 half-life $(12-14 \mathrm{~ms})$ at 12,18 , and $24 \mathrm{~h}$ of incubation at $37^{\circ} \mathrm{C}$.

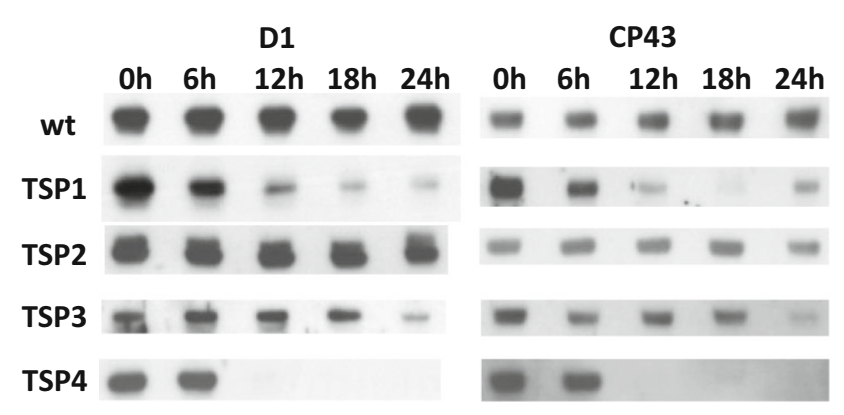

Fig. 3 Immunoblots of PSII subunits, D1 and CP43, in C. reinhardtii cultures taken at different time points of adaptation to $37^{\circ} \mathrm{C}$. Wildtype (wt) and four mutant (TSP1, TSP2, TSP3, and TSP4) samples were assayed with antibodies against D1 (left) and CP43 (right). The amounts of sample loaded corresponded to $1.4 \mu \mathrm{g}$ chlorophyll 
We performed immunoblotting analyses to evaluate the levels of the PSII subunits, D1 and CP43, in each microalgae strain (Fig. 3). Wild-type and TSP2 cells maintained equal amounts of D1 and CP43 over the entire period of incubation. However, in TSP1 and TSP3, D1 and CP43 decreased after 12 and $24 \mathrm{~h}$ of incubation, respectively. In TSP4, both subunits were completely absent after $12 \mathrm{~h}$ of incubation.

To determine whether these mutants were capable of producing $\mathrm{H}_{2}$ and whether they could sustain $\mathrm{H}_{2}$ production over long time periods, the cultures were transferred to sealed, initially anaerobic flasks, and incubated in the light and in the dark. At different time points, the gas in the headspace of the flasks was measured with a gas chromatograph (GC). At $25{ }^{\circ} \mathrm{C}$ in the light, the wild-type and all four mutants accumulated oxygen in the flasks, and no significant hydrogen accumulation was detected. At $37{ }^{\circ} \mathrm{C}$ in the dark, no oxygen accumulated, and all cultures accumulated equal amounts of hydrogen over $24 \mathrm{~h}$. The hydrogen production rate was $0.13 \mathrm{nmol} \mu \mathrm{g}^{-1} \mathrm{~h}^{-1}$. At $37{ }^{\circ} \mathrm{C}$ in the light, wild-type cultures accumulated oxygen, and no continuous hydrogen accumulation was detected. After $24 \mathrm{~h}, 30 \mathrm{nmol}$ of hydrogen per $\mathrm{ml}$ culture was measured, and no significant increase was detected thereafter. Conversely, the mutants did not accumulate any oxygen, but accumulated continuously hydrogen (Fig. 4a). During 3 days, the mutants TSP1, TSP2, TSP3, and TSP4 accumulated 850, 5330, 1340, and $170 \mathrm{nmol}$ hydrogen per $\mathrm{ml}$ of culture, respectively. If supplemented with the PSII inhibitor DCMU, all cultures including the wild type accumulated hydrogen continuously in the light. During 3 days, they produced $170-450 \mathrm{nmol}$ hydrogen per $\mathrm{ml}$ culture. The average hydrogen production rate during the most productive day was calculated for all hydrogen-producing cultures (Fig. 4b). With no DCMU, these values were $3.1,11.7,3.9$, and $0.5 \mathrm{nmol} \mu \mathrm{g}^{-1} \mathrm{~h}^{-1}$ for mutants TSP1, TSP2, TSP3, and TSP4, respectively. If supplemented with DCMU, the values of wild-type and mutant cultures were comparable and ranged between 0.5 and $1.5 \mathrm{nmol} \mu \mathrm{g}^{-1} \mathrm{~h}^{-1}$.

Next, we tested mutant recovery to the wild-type phenotype. After 24 -h incubations at $37{ }^{\circ} \mathrm{C}$, mutant cultures were transferred back to $25^{\circ} \mathrm{C}$. Analogous measurements of OER, PSII fluorescence, and P700 absorbance were performed over a period of $24 \mathrm{~h}$ (Fig. 5). TSP1, TSP2, and TSP4 recovered steadily over the entire period; they reached 82,100 , and $64 \%$ of wild-type OER values, respectively, after $24 \mathrm{~h}$. TSP3 reached wild-type OER values after $6 \mathrm{~h}$ of incubation at $25^{\circ} \mathrm{C}$. Additionally, TSP 1 and TSP4 reached 80 and $45 \%$, respectively, of wild-type PSII quantum efficiency. Furthermore, TSP4 fully recovered the oxidized P700 half-life of wild-type microalgae.
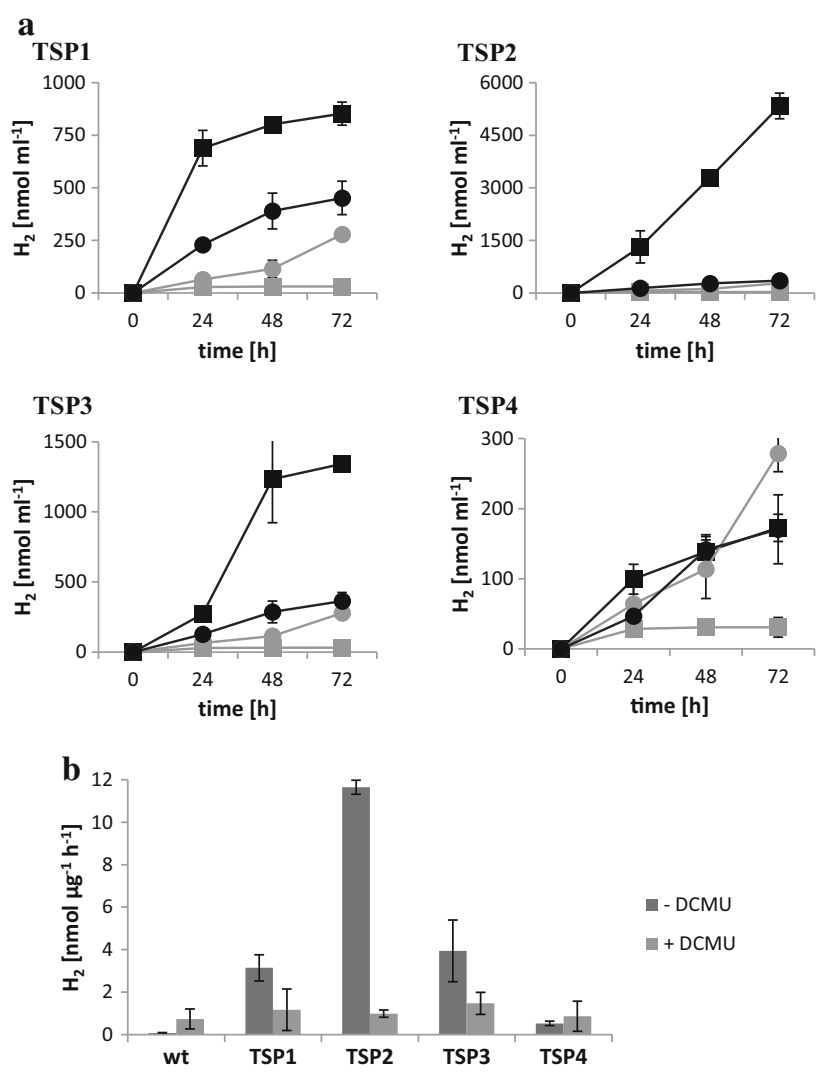

Fig. 4 Hydrogen production in the light at $37^{\circ} \mathrm{C}$. a Hydrogen accumulation in sealed flasks per ml culture over 3 days. In all charts, the gray curves correspond to the wild type; the black curves correspond to TSP1, TSP2, TSP3, and TSP4, as indicated. The squares and circles represent cultures without and with DCMU, respectively. b Average hydrogen production rates during the most productive day. The values were normalized to chlorophyll content. The dark and light bars represent cultures without and with DCMU, respectively. All cultures were grown at $25{ }^{\circ} \mathrm{C}$, incubated for 1 day at $37^{\circ} \mathrm{C}$, and then transferred to sealed bottles without and with $10 \mathrm{mM}$ DCMU. The light intensity was $60-70 \mu \mathrm{E} \mathrm{m}^{-2} \mathrm{~s}^{-1}$ during growth and incubation at $37^{\circ} \mathrm{C}$. Data represent the mean \pm SD of four independent experiments

\section{Toward a photosynthetic cyclic hydrogen-producing bioreactor}

To fully assess the relevance of the presented mutants, their hydrogen production capability needs to be characterized in a cyclic bioreactor (Fig. 6). An important feature of our proposed hydrogen-producing bioreactor was the ability to exchange gases, particularly hydrogen, from the aqueous culture to the gas phase of the acclimated chamber. Therefore, we tested hydrogen production and diffusion through silicon tubes in a prototype bioreactor consisting only of the anaerobic hydrogen production chamber. The mutant TSP2 was adapted to $37{ }^{\circ} \mathrm{C}$ for $24 \mathrm{~h}$ and transferred to a sealed, anaerobic bottle. The adapted culture was pumped through the prototype bioreactor. The mutant 

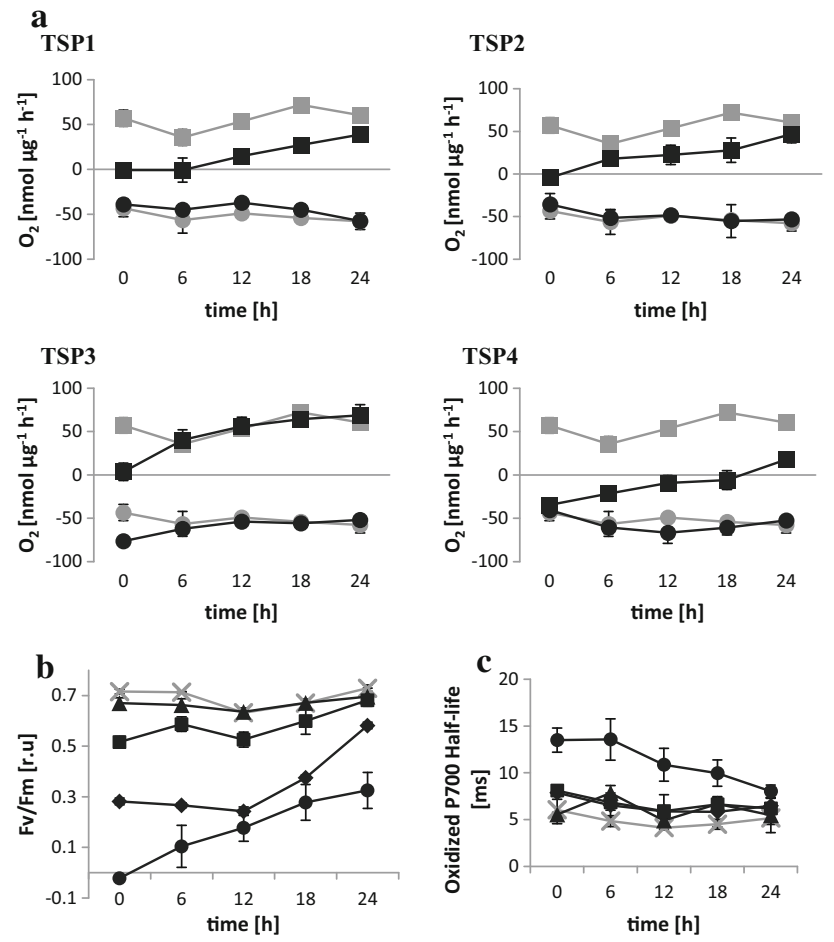

Fig. 5 Physiological measurements of $C$. reinhardtii wild-type (gray) and TSP mutant (black) cultures during recovery at $25^{\circ} \mathrm{C}$, after 24-h incubations at $37{ }^{\circ} \mathrm{C}$. a Oxygen exchange rate in the dark (circles) and in the light at $300 \mu \mathrm{E} \mathrm{m}^{-2} \mathrm{~s}^{-1}$ (squares); $\mathbf{b}$ maximum quantum efficiency of PSII; c oxidized P700 half-life. In b and c, TSP1, TSP2, TSP3, and TSP4 are represented by diamonds, triangles, squares, and circles, respectively. The light intensity was $60-70 \mu \mathrm{E} \mathrm{m}^{-2} \mathrm{~s}^{-1}$ during growth, at $37^{\circ} \mathrm{C}$, and during recovery at $25^{\circ} \mathrm{C}$. Data represent the mean $\pm \mathrm{SD}$ of three independent experiments

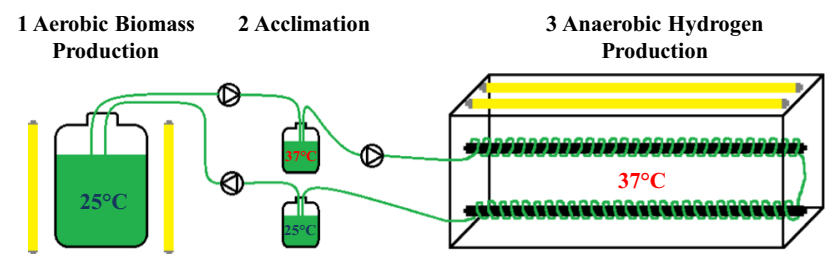

Fig. 6 Cyclic photosynthetic hydrogen-producing bioreactor. The microalgal culture circulates between two illuminated main chambers. At $25{ }^{\circ} \mathrm{C}$, the culture grows in an aerobic bottle $(1)$; at $37{ }^{\circ} \mathrm{C}$, the culture produces hydrogen in silicon tubing which passes through a sealed anaerobic collection chamber (3). While cycling from one chamber to the other, the culture is acclimated to the required temperature (2)

could produce hydrogen in the silicon tubes, which diffused immediately into the anaerobic chamber, at the same rate achieved in a sealed bottle. This experiment set the stage for the design and implementation of a cyclic photosynthetic hydrogen-producing bioreactor, as we previously proposed (Mazor et al. 2012).

\section{Discussion}

We identified four mutant microalgae strains that, compared to wild-type microalgae, exhibited a faster rate of decline in the ability to evolve oxygen, upon incubation at $37{ }^{\circ} \mathrm{C}$. In contrast, these mutants maintained oxygen respiration at wild-type levels. Before the end of the 24-h incubation, all mutants, but no wild-type cultures, reached a point where oxygen uptake by respiration was higher than oxygen production. Thus, mutants exhibited net oxygen consumption in the light.

The net oxygen consumption condition is absolutely necessary for our proposed system to maintain anoxic conditions and enable hydrogen production. In fact, at $37^{\circ} \mathrm{C}$, all four mutants evolved hydrogen continuously in the light, over the period of 3 days. The average hydrogen production rates during the most productive day ranged between 0.5 and $11.7 \mathrm{nmol} \mu \mathrm{g}^{-1} \mathrm{~h}^{-1}$. With the addition of DCMU or in the dark, the hydrogen production rates were $0.5-1.5$ or $0.13 \mathrm{nmol} \mu \mathrm{g}^{-1} \mathrm{~h}^{-1}$ in all mutant and wild-type cultures. Hence, the observed hydrogen production in the mutant cultures was mainly light dependent, and the hydrogenase received electrons through the photosynthetic ETC from residual PSII activity as well as from other sources. However, we observed different amounts of lightdependent hydrogen production among the various mutants, mainly due to a differential residual PSII contribution. This residual PSII activity raises the question of which reducing equivalent source was used for respiration to sustain anoxic conditions in the mutants. Acetate present in the media might have contributed to respiration. However, an acetate independent respiration would be desirable and starch accumulation remains to be elucidated.

Each of the selected TSP mutants exhibited unique properties. They showed different PSII inactivation time courses, different changes in the amounts of PSII subunits, and different levels of oxygen evolution recovery upon return to the permissive temperature. Mutant TSP1 exhibited a reduced ability to evolve oxygen in light at $37^{\circ} \mathrm{C}$ over the entire incubation time. In addition, PSII fluorescence decreased to $60 \%$ of wild-type levels, and D1 and CP43 also decreased to minimal amounts. The oxidized half-life of P700 was unaltered, which suggested that, despite the lower PSII activity, these cultures maintained electron transfer to PSI. However, the amount of P700 was $30 \%$ lower than that in wild-type cultures. Nevertheless, PSII independent hydrogen production reached the highest value in this mutant, suggesting that PSI is not a limiting factor. Still, residual PSII activity was the main contributor for hydrogen production, at a lower rate compared to the TSP2 and TSP3 mutants. 
In the TSP2 mutant, the OER decreased steadily, from 6 to $24 \mathrm{~h}$ of incubation at $37{ }^{\circ} \mathrm{C}$, until it reached around $0 \mathrm{nmol} \mu \mathrm{g}^{-1} \mathrm{~h}^{-1}$, which was maintained thereafter. This scenario is optimal for our proposed system. Water oxidation continued to provide electrons for hydrogen production, but not at a rate that could compromise the maintenance of the required anoxic conditions. Fluorescence measurements and the detection of D1 and CP43 suggested that at least the core complex of PSII was not damaged at any point during the incubation. Furthermore, P700 levels are like in the wild type. These optimal conditions gave rise to the highest observed hydrogen production rate: $11.7 \mathrm{nmol} \mu \mathrm{g}^{-1} \mathrm{~h}^{-1}$.

In the TSP3 mutant, the OER decreased steadily, as observed in the TSP2 mutant, until it reached an OER of around $0 \mathrm{nmol} \mu \mathrm{g}^{-1} \mathrm{~h}^{-1}$ at $24 \mathrm{~h}$; thereafter it decreased only minimally. PSII fluorescence remained at wild-type levels up to $18 \mathrm{~h}$ of incubation, and then declined by $25 \%$ at $24 \mathrm{~h}$. Immunoblots revealed reduced D1 and CP43 levels at $24 \mathrm{~h}$ of incubation. TSP3 had 20-40 \% higher amounts of P700 than wild-type microalgae, over the period of incubation, and they exhibited the same half-life for oxidized P700. The higher amounts of P700 did not increase significantly the PSII independent hydrogen production rate, suggesting again that P700 is not a limiting factor. As well in this mutant, PSII was the main contributor for hydrogen production. The productivity was somewhat higher than in the TSP1 mutant and quite lower than in the TSP2 mutant. This suggests a further decline of PSII after the adaptation time of $24 \mathrm{~h}$.

In the TSP4 mutant, the OER decreased to almost $0 \mathrm{nmol} \mu \mathrm{g}^{-1} \mathrm{~h}^{-1}$ after only $6 \mathrm{~h}$ of incubation; after $12 \mathrm{~h}$, oxygen production was completely absent. PSII fluorescence was also completely absent, starting at $12 \mathrm{~h}$ and continuing throughout the study period. D1 and CP43 levels were practically undetectable on immunoblots. This most severe phenotype had also an effect on P700 reduction. After $12 \mathrm{~h}$ of incubation, the half-life of oxidized P700 was 12-14 ms, or about twice the half-life observed in wild-type microalgae. Furthermore, the amount of P700 decreased by $30 \%$ after $12 \mathrm{~h}$ of incubation. These features resulted in a relatively low hydrogen production rate $\left(0.5 \mathrm{nmol} \mu \mathrm{g}^{-1} \mathrm{~h}^{-1}\right)$. Nevertheless, this rate was higher than the rate achieved in the dark. Hence, although no electrons could be delivered from water oxidation, PSI delivered electrons for hydrogen production.

A crucial element in our proposed cyclic hydrogen production system is the ability of mutants to return to the wild-type photosynthetic phenotype after a cycle of hydrogen production. This feature was achieved in all four mutants; after $24 \mathrm{~h}$ at $37{ }^{\circ} \mathrm{C}$, all mutants recovered the wild-type phenotype upon incubation at $25{ }^{\circ} \mathrm{C}$.
Several previous studies were conducted to establish an economically viable method for large-scale photosynthetic hydrogen production systems. Many focused on generating an $\mathrm{O}_{2}$-insensitive hydrogenase (Bandyopadhyay et al. 2010; Burgess et al. 2011). That system would be beneficial, because it includes PSII to supply a maximal amount of electrons for hydrogen production. However, no significant decrease in $\mathrm{O}_{2}$ sensitivity has been achieved; furthermore, that system would not include the necessary spatial separation of oxygen and hydrogen to prevent combustion.

The most common protocol for achieving sustained $\mathrm{H}_{2}$ production in the light was sulfur starvation (Melis et al. 2000; Melis and Happe 2001). In that protocol, cultures are grown normally under aerobic conditions, and then they are centrifuged and resuspended in sulfur-depleted media. Upon sulfur deprivation, cells are unable to synthesize proteins that contain sulfur, like D1. Because the D1 protein of PSII has a short half-life, it must be continuously replaced by newly synthesized D1 to ensure PSII activity. Therefore, this protocol causes a reduction in PSII activity over the first 20-30 h, but oxygen respiration is maintained (Kosourov et al. 2002). As a result, anoxic conditions are reached in a closed environment, and hydrogen production takes place over several days, with maximal hydrogen production rates of $4-6 \mathrm{nmol} \mu \mathrm{g}^{-1} \mathrm{~h}^{-1}$ under standard conditions and up to $9.4 \mathrm{nmol} \mu \mathrm{g}^{-1} \mathrm{~h}^{-1}$ under optimized growth conditions (Kosourov et al. 2003). Eventually, the cells die from sulfur starvation. In that system, the reduction in PSII activity causes a reduction in energy conversion efficiency; however, it enables sustained hydrogen production over a long period of time.

It has been calculated that biophotolytic energy conversion efficiencies must be around $5 \%$ for economic viability (Kruse et al. 2005); currently, efficiencies of around $1 \%$ have been achieved with sulfur deprivation (Kruse et al. 2005). Many different approaches to the sulfur starvation system have been investigated in endeavors to increase hydrogen production efficiencies (Burgess et al. 2011). Those studies raised the hope that $5 \%$ energy conversion efficiencies might be achievable in that system. However, the system may not be realizable at large scales, due to the impracticality of transferring cells into sulfurdepleted media. Furthermore, the cell death caused by sulfur starvation limits this system to a batch process. Therefore, direct regulation of PSII in a controlled, simple manner would be more practical in an analogous system. Rochaix and colleagues attempted the latter approach with an inducible promoter. That protocol proved to be an effective method for controlling hydrogen production without inducing fatal stress to the cells; thus, cyclic hydrogen production is achievable in that system (Surzycki et al. 2007). However, the promotor was induced by 
copper; therefore, cell transfer between copper-containing and copper-free media remains an obstacle for upscaling the protocol.

The present study described a system that employed TSP mutants and induction of anoxic conditions by increasing the incubation temperature. These conditions triggered hydrogen production at the same rates achieved by the sulfur starvation protocol. However in our system, cells were not exposed to deadly stress, and they could be recycled by simply reducing the temperature. Thus, these TSP mutants provide a convenient platform for increasing hydrogen production by physical means to achieve $5 \%$ energy conversion efficiencies in a platform that is amenable to upscaling.

The upscale system would require two compartments: one for accumulating biomass and the other for producing hydrogen. The first compartment could be an open pond, which is currently used industrially to grow Dunaliella for producing B-carotene (Ben-Amotz et al. 1989; Oren 2014). The second compartment should be a tubular reactor composed of transparent, H2-permeable tubing. This reactor must be enclosed in a translucent, airtight chamber. The second compartment was successfully tested, on a small scale, in the present study. In the absence of air convection, the temperature could be increased in the second compartment without an external energy source; this feature could facilitate the development of the mutant phenotype and allow efficient hydrogen production. Hydrogen then could diffuse through the tubing and accumulate in the airtight compartment for collection.

\section{Conclusion}

In this work, we created TSP mutants of $C$. reinhardtii that could alternate between oxygenic photosynthesis and anoxic hydrogen production, in response to changes in temperature. This approach represented a solution to the challenge posed by the oxygen sensitivity of the [FeFe]hydrogenase. Our protocol was an analogous, however viable, alternative to the sulfur starvation protocol for sustained hydrogen production. Consequently, these mutants are an adequate system for upscaling sustained hydrogen production. Studies that aim to increase energy conversion efficiencies in sulfur-starved cells might be tested with these TSP mutants. Therefore, they represent an alternative platform for overcoming the obstacle of noneconomically viable energy conversion efficiencies. Finally, the proven quality of $C$. reinhardtii as a model organism for industrial endeavors provides confidence that the developed technology can be readily transferred to a mass hydrogen production plant.
Acknowledgments This work was supported by grants from the European Research Council-ERC (No 293579-HOPSEP) and from the Israel Science Foundation (ISF Grant 71-14).

Open Access This article is distributed under the terms of the Creative Commons Attribution 4.0 International License (http://crea tivecommons.org/licenses/by/4.0/), which permits unrestricted use, distribution, and reproduction in any medium, provided you give appropriate credit to the original author(s) and the source, provide a link to the Creative Commons license, and indicate if changes were made.

\section{References}

Amon DI, Mitsui A, Paneque A (1961) Photoproduction of hydrogen gas coupled with photophosphorylation. Science 134:1425

Amunts A, Nelson N (2009) Plant photosystem I design in light of evolution. Structure 17:637-650

Arnon DI (1949) Copper enzymes in isolated chloroplasts. Polyphenoloxidase in Beta vulgaris. Plant Physiol 24:1-15

Baker NR (2008) Chlorophyll fluorescence: a probe of photosynthesis in vivo. Annu Rev Plant Biol 59:89-113

Bandyopadhyay A, Stöckel J, Min H, Sherman LA, Pakrasi HB (2010) High rates of photobiological $\mathrm{H} 2$ production by a cyanobacterium under aerobic conditions. Nat Commun 1:139

Barber J (2004) Engine of life and big bang of evolution: a personal perspective. Photosynth Res 80:137-155

Ben-Amotz A, Shaish A, Avron M (1989) Mode of action of the massively accumulated beta-carotene of Dunaliella bardawil in protecting the alga against damage by excess irradiation. Plant Physiol 91:1040-1043

Bendall DS, Howe CJ, Nisbet EG, Nisbet RE (2008) Photosynthetic and atmospheric evolution. Philos Trans R Soc Lond B Biol Sci 363:2625-2628

Blankenship RE, Hartman H (1998) The origin and evolution of oxygenic photosynthesis. Trends Biochem Sci 23:94-97

Burgess SJ, Tamburic B, Zemichael F, Hellgardt K, Nixon PJ (2011) Solar-driven hydrogen production in green algae. Adv Appl Microbiol 75:71-110

Falkowski PG, Isozaki Y (2008) The story of O2. Science 322:540-542

Florin L, Tsokoglou A, Happe T (2001) A novel type of iron hydrogenase in the green alga Scenedesmus obliquus is linked to the photosynthetic electron transport chain. J Biol Chem 276:6125-6132

Gest H, Kamen MD (1949) Photoproduction of molecular hydrogen by Rhodospirillum rubrum. Science 3:558-559

Ghirardi ML, Togasaki RK, Seibert M (1997) Oxygen sensitivity of algal H2-production. Appl Biochem Biotechnol 63-65:141-151

Happe T, Kaminski A (2002) Differential regulation of the Fehydrogenase during anaerobic adaptation in the green alga Chlamydomonas reinhardtii. Eur J Biochem 269:1022-1032

Harris EH (2008) The Chlamydomonas sourcebook, 2nd edn. Elsevier, Amsterdam

Hemschemeier A, Fouchard S, Cournac L, Peltier G, Happe T (2008) Hydrogen production by Chlamydomonas reinhardtii: an elaborate interplay of electron sources and sinks. Planta 227:397-407

Kosourov S, Tsygankov A, Seibert M, Ghirardi ML (2002) Sustained hydrogen photoproduction by Chlamydomonas reinhardtii: effects of culture parameters. Biotechnol Bioeng 78:731-740

Kosourov S, Seibert M, Ghirardi ML (2003) Effects of extracellular $\mathrm{pH}$ on the metabolic pathways in sulfur-deprived, H2-producing Chlamydomonas reinhardtii cultures. Plant Cell Physiol 44(2):146-155 
Kruse O, Hankamer B (2010) Microalgal hydrogen production. Curr Opin Biotechnol 21:238-243

Kruse O, Rupprecht J, Mussgnug JH, Dismukes GC, Hankamer B (2005) Photosynthesis: a blueprint for solar energy capture and biohydrogen production technologies. Photochem Photobiol Sci 4:957-970

Lambert GR, Smith GD (1977) Hydrogen formation by marine Bluegreen algae. FEBS Lett 83:159-162

Liveanu V, Yocum CF, Nelson N (1986) Polypeptides of the oxygenevolving photosystem II complex; immunological detection and biogenesis. J. Biol Chem 26(1):5296-5300

Mazor Y, Toporik H, Nelson N (2012) Temperature-sensitive PSII and promiscuous PSI as a possible solution for sustainable photosynthetic hydrogen production. Biochim Biophys Acta 1817:1122-1126

Melis A, Happe T (2001) Hydrogen production. Green Algae as a source of energy. Plant Physiol 127:740-748

Melis A, Zhang L, Forestier M, Ghirardi ML, Seibert M (2000) Sustained photobiological hydrogen gas production upon reversible inactivation of oxygen evolution in the green alga Chlamydomonas reinhardtii. Plant Physiol 122:127-136

Nelson N (2011) Photosystems and global effects of oxygenic photosynthesis. Biochim Biophys Acta 1807:856-863

Nelson N, Ben-Shem A (2004) The complex architecture of oxygenic photosynthesis. Nat Rev Mol Cell Biol 5:971-982
Nelson N, Junge W (2015) Structure and energy transfer in photosystems of oxygenic photosynthesis. Annu Rev Biochem 84:659-683

Nelson N, Yocum C (2006) Structure and function of photosystems I and II. Annu Rev Plant Biol 57:521-565

Oren A (2014) The ecology of Dunaliella in high-salt environments. J Biol Res (Thessalon) 21:23

Redding K, Cournac L, Vassiliev IR, Golbeck JH, Peltier G, Rochaix JD (1999) Photosystem I is indispensable for photoautotrophic growth, $\mathrm{CO}_{2}$ fixation, and $\mathrm{H} 2$ photoproduction in Chlamydomonas reinhardtii. J Biol Chem 274:10466-10473

Stuart TS, Gaffron H (1972) The mechanism of hydrogen photoproduction by several algae. II. The contribution of photosystem II. Planta (Berlin) 106:101-112

Surzycki R, Cournac L, Peltier G, Rochaix JD (2007) Potential for hydrogen production with inducible chloroplast gene expression in Chlamydomonas. Proc Natl Acad Sci USA 104:17548-17553

Winkler M, Kuhlgert S, Hippler M, Happe T (2009) Characterization of the key step for light-driven hydrogen evolution in green algae. J Biol Chem 284:36620-36627

Yacoby I, Pochekailov S, Toporik H, Ghirardi ML, King PW, Zhang S (2011) Photosynthetic electron partitioning between [FeFe]hydrogenase and ferredoxin: NADP+- oxidoreductase (FNR) enzymes in vitro. PNAS 108:9396-9401 